\title{
Loss of E-cadherin promotes prostate cancer metastasis via upregulation of metastasis-associated gene 1 expression
}

\author{
LIANGSHENG FAN $^{1 *}$, HONGYAN WANG $^{1 *}$, XI XIA $^{2}$, YUMEI RAO $^{3}$, \\ XIANGYI MA ${ }^{1}$, DING MA ${ }^{1}$, PENG WU ${ }^{1}$ and GANG CHEN ${ }^{1}$ \\ ${ }^{1}$ Cancer Biology Medical Center, Tongji Hospital, Tongji Medical College, Huazhong University of Science and Technology, \\ Wuhan, Hubei 430030; ${ }^{2}$ Department of Obstetrics and Gynecology, South Mountain Hospital of Guangdong Medical College, \\ Shenzhen, Guangdong 518000; ${ }^{3}$ Department of Obstetrics and Gynecology, \\ The First Affiliated Hospital of Zhengzhou University, Zhengzhou, Henan 450052, P.R. China
}

Received March 28, 2012; Accepted July 9, 2012

DOI: $10.3892 / \mathrm{ol} .2012 .934$

\begin{abstract}
E-cadherin is a key cell-to-cell adhesion molecule associated with the invasion and metastasis of tumor cells; however, the molecular mechanisms are not entirely understood. In this study, we investigated whether downregulation of E-cadherin by E-cadherin-specific small intefering RNA (siRNA) was able to promote malignant phenotypes of prostate cancer cells through upregulating the metastasis-associated gene 1 (MTA1) in vitro. The expression levels of E-cadherin in human paired prostate adenocarcinoma cell lines, PC-3M-2B4 (2B4) and PC-3M-1E8 (1E8), were investigated using western blot analysis. The alteration of malignant phenotypes associated with decreasing E-cadherin expression were assessed in 2B4 cells using wound-healing assays, solid-phase adhesion assays, invasion assays and cytoskeletal staining. The expression of E-cadherin and MTA1 in normal, localized and metastatic prostate cancer cells was analyzed using immunohistochemistry. Downregulation of E-cadherin using an RNA interference approach led to the upregulation of MTA1 expression, decreased tumor cell adhesion ability as well as enhanced cell mobility, invasion and cellular polarity compared with the controls $(\mathrm{P}<0.05)$. E-cadherin regulated MTA1 in a time-dependent manner. The correlation between E-cadherin and MTA1 was inversed in the prostate cancer group $\left(\mathrm{P}<0.05 ; \mathrm{r}_{\mathrm{s}}=-0.434\right)$. The data suggest that E-cadherin plays an important role in prostate cancer metastasis, which is likely to be due to the regulation
\end{abstract}

Correspondence to: Dr Peng Wu or Dr Gang Chen, Cancer Biology Medical Center, Tongji Hospital, Tongji Medical College, Huazhong University of Science and Technology, 1095 Jiefang Avenue, Wuhan, Hubei 430030, P.R. China

E-mail: pengwu8626@yahoo.com.cn

E-mail: gumpc@126.com

*Contributed equally

Key words: E-cadherin, metastasis-associated gene 1, prostate carcinoma, metastasis of MTA1 expression. E-cadherin may combine with MTA1 and alter the malignant phenotype of prostate cancer cells. A combined testing strategy for detecting MTA1 and E-cadherin may be sufficient for selecting high-risk patients with metastasis. Therefore, E-cadherin and MTA1 may be potential powerful factors for the treatment of various types of cancer.

\section{Introduction}

At present, prostate cancer is recognized as one of the most important medical problems facing the male population. In the USA and Europe, prostate cancer is currently the second most common cause of cancer mortality in males $(1,2)$. The latest statistics reveal that among males in the USA, an estimated 240,890 new cancer cases and 33,720 mortalities due to prostate cancer occurred in 2011 (1). Prostate cancer-related mortality is largely due to its high metastatic potential for bone and/or other organs $(3,4)$. Clinically, the prevention and treatment of prostate cancer metastasis remains a significant challenge since the molecular mechanisms of prostate cancer invasion and metastasis are not well understood.

Metastasis is a complex and multi-step process in the progression of malignant cancer (5). Cell migration results in the spreading of cancer, which is the leading cause of cancer-related mortalities. In the process of cancer progression, certain cell adhesion molecules (CAMs) play a pivotal role in the development of recurrent, invasive and distant metastasis (6). A loss or reduction in the expression of CAMs, including cadherins, facilitates the detachment of single cancer cells from the tumor bulk $(7,8)$. One of the key molecules critical for cell-to-cell adhesion is E-cadherin, a membrane glycoprotein located at cell adherent junctions $(9,10)$. E-cadherin aids the assembly of epithelial cells and maintains the quiescence of cells within sheets by forming adherent junctions with adjacent epithelial cells (11). A number of studies have demonstrated that increased expression of E-cadherin is able to inhibit invasion and metastasis, while a reduced expression potentiates these phenotypes (11-14). In order for epithelial cells to develop into cancer cells, activation of the epithelial-mesenchymal transition (EMT) is required (15). EMT causes the epithelial 
cell layers to lose polarity and cell-cell contacts. It therefore triggers the remodeling of the cellular skeleton $(16,17)$. Upregulation of E-cadherin is implicated by the activation of EMT $(14,18)$, and E-cadherin is regarded as a main indicator of epithelial/mesenchymal phenotype switching (19).

The metastasis-associated gene 1 (MTA1) was originally identified by the differential screening of a cDNA screening library using highly metastatic mammary adenocarcinoma cell lines (20). MTA1 appears to interact with, or may even be a member of, the histone deacetylase (HDAC) complex, and acts as a co-activator of this complex (21). Studies have demonstrated that MTA1 overexpression is associated with the adhesion, invasion and metastasis of certain cancer cells (22-24), and with a higher tumor grade, the development, microvascular invasion and poor prognosis in a number of malignant cancer types (25). Through repression of the estrogen receptor $\alpha(\mathrm{ER} \alpha)$, hypoxia-inducible factor- $1 \alpha(\mathrm{HIF}-1 \alpha)$ and p53 protein, MTA1 converts cancer cells into a more aggressive phenotype (26). Moreover, MTA1 has been identified to determine EMT phenotypes mainly through downregulating the expression of E-cadherin, which leads to EMT $(27,28)$. E-cadherin can be upregulated using MTA1 small interfering RNA (siRNA) in melanoma cells, which was also confirmed in our previous study in cervical cancer cells $(29,30)$. MTA1 and E-cadherin are involved in the EMT process (28), since the loss of E-cadherin expression has been demonstrated to increase cancer metastasis progresses $(13,14,31)$, and tumor cells with increased expression of MTA1 indicate more invasive phenotypes (32). Negative feedback regulation is crucial for cells to determine their fate and maintain function during gene regulation. Our other study (unpublished data) provided information concerning the regulation of E-cadherin expression by MTA1 when controlling malignant phenotypes in prostate cancer. Whether E-cadherin has an effect on MTA1 expression has not yet been elucidated.

In the present study, we examined whether the expression of MTA1 is an important contributing factor to the metastasis induced by E-cadherin loss in vitro. In addition, we investigated the correlation between E-cadherin and MTA1 expression and location in prostate cancer and metastatic prostate cancer tissue samples. We identified that loss of E-cadherin expression changes the malignant phenotype of prostate cancer cells through an MTA1-dependent pathway.

\section{Materials and methods}

Reagents and antibodies. All reagents were of analytical grade and commercially purchased. Primary antibodies against MTA1 were from Santa Cruz Biotechnology, Inc. (Santa Cruz, CA, USA). E-cadherin was obtained from Epitomics Inc. (Burlingame,CA,USA). $\alpha$-tublin, $\beta$-actin and alkaline phosphatase-conjugated anti-rabbit/mouse/goat IgGs were purchased from Sigma (Deisenhofen, Germany). FITC/Cy3-conjugated IgG was obtained from Proteintech Group Inc. (Chicago, IL, USA). 4,6-Diamino-2-pheylindoledi (DAPI), fibronectin (FN) and 3-(4,5-dimethylthiazol-2-yl)-2,5-diphenyltetrazolium bromide (MTT) were purchased from Sigma. The SP histostain-plus kit was obtained from ZhongShan Biotech Co. (Beijing, China). All other chemicals were of analytical grade and obtained from standard suppliers.
Cell lines. The human paired poorly-metastatic prostate adenocarcinoma cell lines PC-3M-2B4 (2B4) and highly-metastatic prostate adenocarcinoma cell lines PC-3M-1E8 (1E8) were kindly provided by Dr Jie Zheng (Beijing University, Beijing, China) (33). All cells were cultured in RPMI-1640 medium (Invitrogen Life Technologies, Carlsbad, CA, USA) with 10\% (v/v) fetal bovine serum (FBS; Sijiqing Co., Hangzhou, China), $100 \mathrm{U} / \mathrm{ml}$ penicillin and $100 \mu \mathrm{g} / \mathrm{ml}$ streptomycin at $37^{\circ} \mathrm{C}$ and $5 \%$ $\mathrm{CO}_{2}$ using a humidified incubator (Heraeus, Hanau, Germany).

Western blot analysis. For western blot analysis, cells were lysed in RIPA buffer containing $50 \mathrm{mM}$ Tris/ $\mathrm{HCl}(\mathrm{pH} 7.2)$, $150 \mathrm{mM} \mathrm{NaCl}, 1 \% \mathrm{NP}-40,0.1 \%$ SDS and $0.5 \%$ (w/v) sodium deoxycholate. Equivalent amounts of cell extracts $(150 \mu \mathrm{g})$ were separated on 10\% SDS-polyacrylamide gel (SDS-PAGE) and transferred onto a polyvinylidene difluoride membrane (PVDF). Filters were blocked in $25 \mathrm{mM}$ Tris ( $\mathrm{pH}$ 8.0) containing $125 \mathrm{mM} \mathrm{NaCl}, 0.1 \%$ Tween 20 and 5\% skimmed milk for $1 \mathrm{~h}$, and then incubated with the diluted primary antibody (MTA1, 1:200; E-cadherin, 1:2000; $\beta$-actin, 1:1000) at $4^{\circ} \mathrm{C}$ overnight. After incubation, the secondary antibodies were added (1:1000 dilution). The immunoreactive bands were visualized with alkaline phosphatase and 5-bromo-4-chloro3-indolyl-phosphate/nitro blue tetrazolium (BCIP/NBT).

Cell transfection. Cell lines were cultured in 6-well tissue culture plates and flasks at $37^{\circ} \mathrm{C}$ and $5 \% \mathrm{CO}_{2}$ using a humidified incubator (Heraeus). Cells were transfected with $200 \mathrm{pmol} / \mathrm{ml}$ of siRNA duplex using $5 \mu \mathrm{l}$ Lipofectamine 2000 when cells were $20 \%$ confluent following the manufacturer's instructions of Lipofectamine 2000 (Invitrogen Life Technologies) siRNA treatment. The controls included untransfected cells and transfected cells with a scrambled negative control siRNA (Ruibo Biotechnology Co., Guangzhou, China). For plasmid transfection, $4 \times 10^{5}$ cells/6-well plate were prepared using $4 \mu \mathrm{g}$ of plasmid and $10 \mu \mathrm{l}$ Lipofectamine 2000 per well. The full length PCMV-SPORT6 E-cadherin plasmid was purchased from Yrbio (Hunan, China). It was reconstructed to pcDNA3.1 E-cadherin in our laboratory (Cancer Biology Medical Center, Tongji Hospital) and the empty vector pcDNA3.1 was preserved. Cells were harvested after $48 \mathrm{~h}$ and protein levels were measured using western blotting. E-cadherin siRNA sequences were as follows: forward, 5'-CAGACAAAGACCAGGACU-AdTdT-3'; reverse, 3'-dTdT GUCUGUUUCUGGUCCUGAU-5'.

Wound healing assay. Exponentially growing cells were detached from culture plates by trypsinization and seeded into 6-well plates $\left(1 \times 10^{5}\right.$ cells/well in complete media) with $10 \% \mathrm{FBS}$ in DMEM. The plates were maintained at $37^{\circ} \mathrm{C}$ in $5 \% \mathrm{CO}_{2}$ at saturated humidity in an incubator overnight. The following day, the confluent cell monolayers were converged and scrape-wounded using a micropipette tip. Floating cells were removed after three washes with serum-free medium, while scratched cells were observed under an inverted phase microscope to identify the scratch widths. Cells were cultured in serum-free culture media and images were captured under phase contrast microscopy every $12 \mathrm{~h}$ after wounding. For evaluation of 'wound closure', five randomly selected points along each wound were marked, and the horizontal distance of 
the migrating cells from the initial wound was measured. Each experiment was performed twice or in triplicate. Data collected were as the mean $\pm \mathrm{SD}$. The measurements were obtained by measuring the area of the wound using Image $\mathbf{J}$ software (available from the National Institute of Health website at http://imagej.nih.gov/ij/download/).

Invasion assay. Transwell chambers with polycarbonate membrane filters with 24-well inserts, $6.5 \mathrm{~mm}$ diameter and $8 \mu \mathrm{m}$ pore size (Corning Life Sciences, Corning, NY, USA) were coated with $1.5 \mathrm{mg} / \mathrm{ml}$ Matrigel $^{\mathrm{TM}}$ (BD Biosciences, Franklin Lakes, NJ, USA). The lower chamber was filled with $600 \mu \mathrm{l}$ RPMI-1640 containing 20\% FBS and mouse embryonic fibroblast cell line (NIH 3T3) supernatant, acting as a chemotactic factor (CF), or just DMEM with $1 \%$ fetal calf serum (FCS). A total of $1 \times 10^{4}$ cells $/ 200 \mu 1$ were seeded into the upper compartment and incubated for $48 \mathrm{~h}$ at $37^{\circ} \mathrm{C}$ and $5 \% \mathrm{CO}_{2}$. Following removal of the non-migratory cells from the upper surface of the filter, invasive cells that had migrated through to the lower surface of the filter were fixed in $2.5 \%$ (v/v) glutaraldehyde for $15 \mathrm{~min}$ and stained with crystal violet for $10 \mathrm{~min}$. The number of invasive cells were calculated by counting at least three randomly chosen visual fields at 100-fold magnification (Leica, Solms, Germany). Three independent experiments were conducted for each set.

Solid-phase adhesion assay. Adhesion was determined using an MTT assay. Exponentially growing cells were detached from culture plates by trypsinization. After washing, cells were resuspended in serum-free medium. Equal numbers of cells $\left(4 \times 10^{4}\right.$ cells/well) were seeded into 96 -well plates precoated with $1 \mu \mathrm{g} / \mathrm{ml} \mathrm{FN}$ (Sigma), which proved to be the most evident in pre-experiments (data not shown). All FN-coated wells were compared with cells seeded in $1 \%(\mathrm{w} / \mathrm{v})$ bovine serum albumin (BSA). After $1 \mathrm{~h}$ of incubation at $37^{\circ} \mathrm{C}$, the plate was immersed in PBS containing $1 \mathrm{mmol} / \mathrm{MgCl}_{2}$ to remove non-adherent cells. The adhered cells were then measured using an MTT assay at $490 \mathrm{~nm}$ wavelength. Subsequently, $30 \mu \mathrm{l}$ of MTT $(5 \mathrm{mg} / \mathrm{ml})$ was added $4 \mathrm{~h}$ prior to the end of the incubation period. Following incubation, the samples were centrifuged at $2200 \mathrm{x} g$ for $5 \mathrm{~min}$. Once the supernatant was removed, $150 \mu \mathrm{l}$ of dimethyl sulphoxide (DMSO) was added to resolve the crystals and the optical density (OD) values of each well was measured at $490 \mathrm{~nm}$ with a microplate reader after $15 \mathrm{~min}$. The OD values reflect the proportion of cells adhered to the FN-coated 96-well plate. The rate of adhesion was calculated using the following equation: (the OD value of test/the OD value of control) x 100. All experiments were conducted four times and repeated twice.

Confocal microscopy imaging. For immunofluorescence cytoskeleton staining, $1 \times 10^{4}$ cells were seeded on glass coverslips (13 mm diameter). Once the coverslips were fixated with 4\% paraformaldehyde (Merck, Haar, Germany) in PBS, cell membranes were permeabilized with $0.2 \%$ (v/v) Triton X-100 in PBS, and non-specific binding sites were blocked with $5 \%$ $(\mathrm{w} / \mathrm{v}) \mathrm{BSA}$ in PBS for $30 \mathrm{~min}$ at $37^{\circ} \mathrm{C}$. Treatment with the first antibody ( $\alpha$-tublin, 1:50) was conducted at $4^{\circ} \mathrm{C}$ overnight. Subsequently, the cells were washed three times with cold PBS and incubated with Cy3-conjugated IgG (1:50 dilution) in PBS

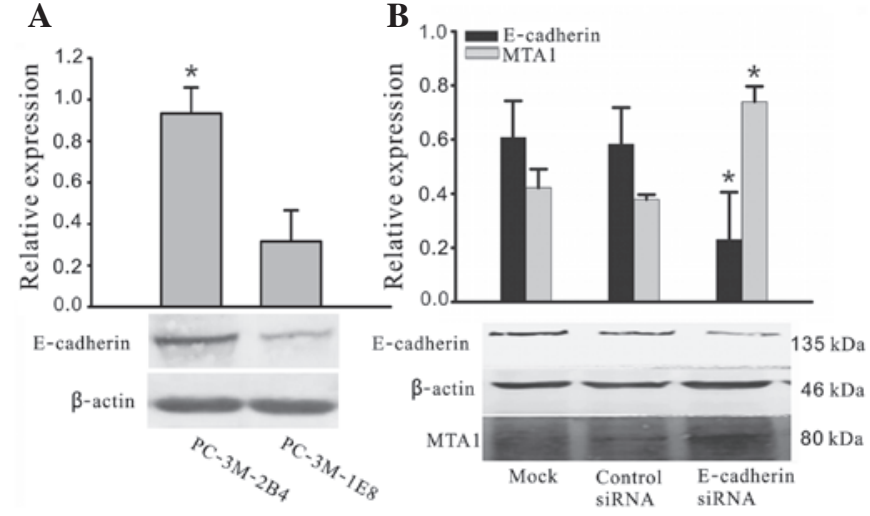

Figure 1. Expression of E-cadherin in prostate carcinoma cell lines. (A) Protein expression levels of E-cadherin in 2B4 cells with poor metastatic ability and 1E8 cells with high metastatic ability were analyzed by western blot analysis using specific antibodies. Expression levels were normalized against $\beta$-actin expression and quantified by bars in three independent experiments. ${ }^{*} \mathrm{P}<0.05$ compared with $1 \mathrm{E} 8$. (B) Cells were treated with E-cadherin siRNA or negative control siRNA for $48 \mathrm{~h}$, and expression of E-cadherin and MTA1 was evaluated by western blot analysis. Expression levels were normalized against $\beta$-actin expression and experiments were repeated three times. E-cadherin siRNA decreased E-cadherin expression and increased MTA1 expression. The quantification of the band intensities is shown. ${ }^{*} \mathrm{P}<0.05$ compared with non-treated cells (mock) and negative control siRNA (control siRNA) transfected cells. MTA1, metastasis-associated gene 1; siRNA, small interfering RNA.

for $30 \mathrm{~min}$ at room temperature. Nuclei were stained for $5 \mathrm{~min}$ at room temperature with DAPI $(2 \mu \mathrm{g} / \mathrm{ml}$ methanol). Cells were then rinsed with PBS and were observed under confocal microscopy (Olympus, Tokyo, Japan).

Immunohistochemistry. Tissues samples of normal prostate as well as localized and metastatic prostate cancer were obtained from the Department of Pathology at Tongji Hospital Affiliated to Huazhong University of Science and Technology (Hubei, China). Immunohistochemical staining for E-cadherin and MTA1 expression were conducted following standard procedures. The $5 \mu \mathrm{m}$ paraffin sections cut on poly-L-lysine-coated microscopy slides were deparaffinized and rehydrated in graded alcohol. The sections were heated in $0.01 \mathrm{M}$ citrate buffer ( $\mathrm{pH} 6.0$ ) in a $85-95^{\circ} \mathrm{C}$ microwave oven ( 3 times for $5 \mathrm{~min}$ each) and the non-specific binding sites were blocked with goat serum. Sections were incubated overnight at $4^{\circ} \mathrm{C}$ with rabbit monoclonal anti-human E-cadherin (1:500 dilution) and goat polyclonal anti-human MTA1 (1:100 dilution) and then washed with PBS. Biotinylated goat anti-rabbit immunoglobulin (Dako, Kyoto, Japan) was then added to the sections for $30 \mathrm{~min}$ at room temperature. Peroxidase-conjugated avidin (Dako) was applied once the sections had been washed with PBS. Peroxidase activity was detected by exposure of the sections to the solution of $0.05 \%$ 3,3'-diaminobenzidine (DAB) and $0.01 \% \mathrm{H}_{2} \mathrm{O}_{2}$ in Tris-HCl buffer (DAB solution) for 3-6 min at room temperature. Finally, the slides were counterstained with hematoxylin. Negative control slides were processed similarly, but were incubated with PBS instead of primary antibody.

Statistical analysis. Data were analyzed using the ANOVA test. Statistical analysis was conducted using SPSS version 13.0 software. $\mathrm{P}<0.05$ was considered to indicate a statistically 
A
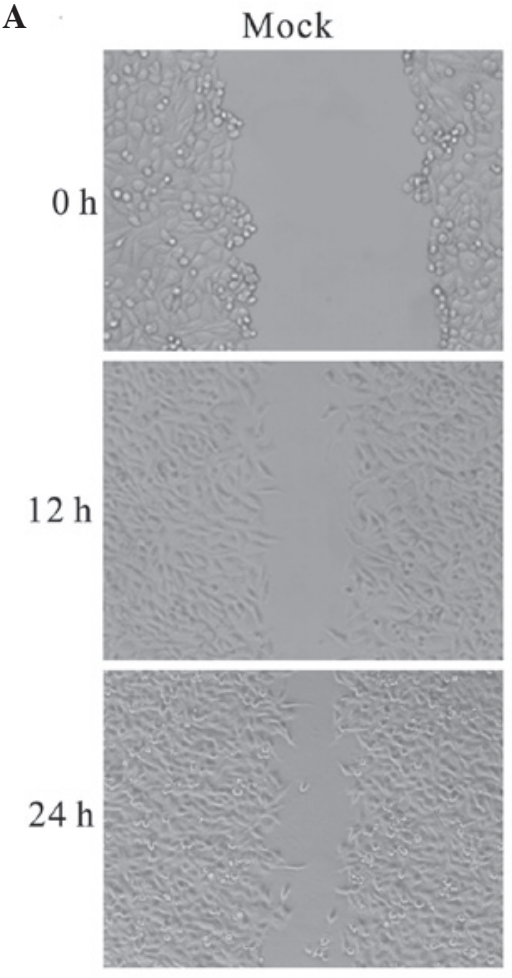

Control siRNA
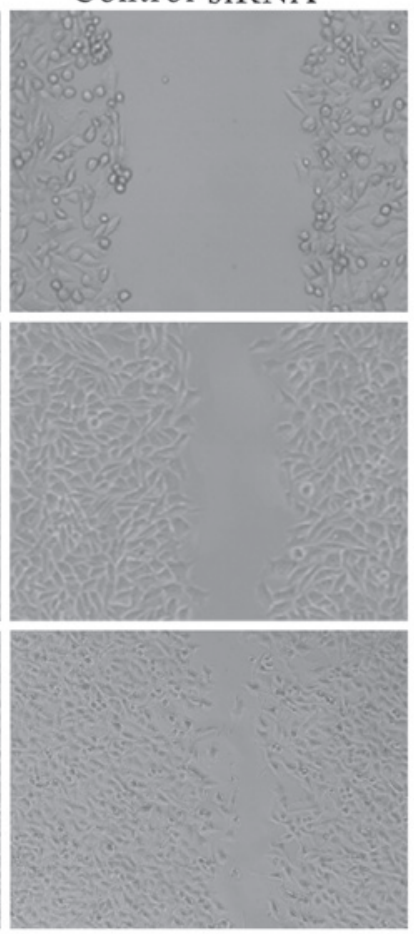

E-cadherin siRNA
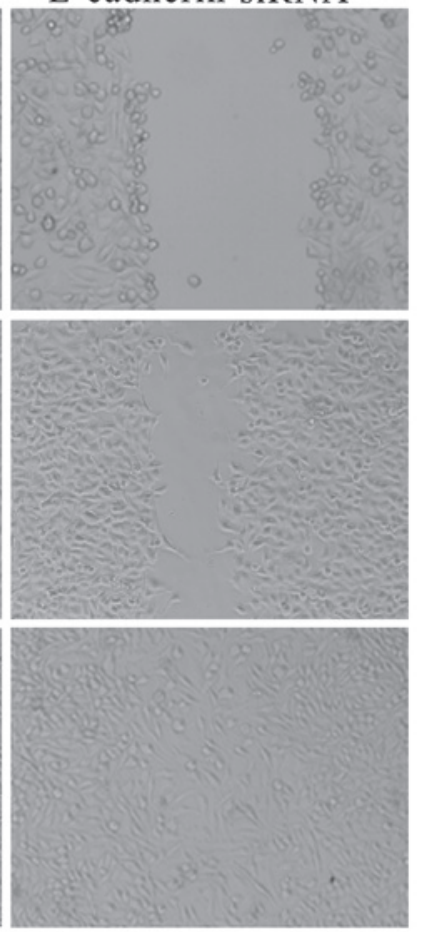

B

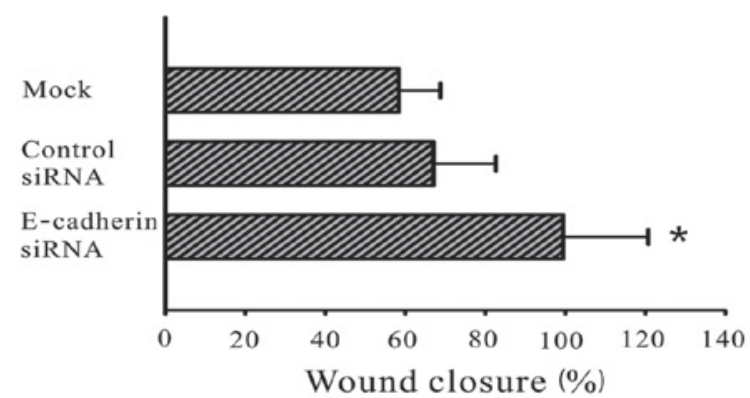

Figure 2. Downregulation of E-cadherin expression increases migration of prostate carcinoma cells. The motility ability was analyzed using a wound healing assay after transfection with E-cadherin siRNA in 2B4 cells for $48 \mathrm{~h}$. Wound closure of scrape-wounded cell monolayers over a $24 \mathrm{~h}$ culture period was assessed. Migration rate (mean \pm SD; $n=3$ ) and degree of wound closure were assessed by measuring the distance between wound edges at $6 \mathrm{~h}$ time intervals. (A) Representative images (magnification, x10) at 0, 12 and $24 \mathrm{~h}$ after wounding from one of three independent experiments are shown. (B) Percentage of wound closure corresponds to the distance between wound edges in at least three randomly chosen regions (mean \pm SD) normalized to $100 \%$ wound closure for E-cadherin siRNA-transfected cells. For each individual experiment, control immunoblots were conducted to ensure E-cadherin inhibition by siRNAs treatments (data not shown). ${ }^{*}<0.001$ between cells transfected with E-cadherin siRNA and mock or control siRNA cells. siRNA, small interfering RNA.

significant difference. The correlation between the indicators was analyzed using Spearman correlation analysis.

\section{Results}

Silencing E-cadherin upregulates MTA1 expression. As shown in Fig. 1A, E-cadherin was present in two prostate cancer cell lines, but at different expression levels. The protein expression levels of E-cadherin in 2B4 cells were over 2.5- to 3-fold higher compared with those in 1E8 cells. Since the 2B4 cells expressed the highest levels of endogenous E-cadherin, we selected this cell line to investigate the effects of heterologous E-cadherin expression on the cellular biological properties of prostate carcinoma cells in vitro. Western blot analysis demonstrated that E-cadherin siRNA is able to specifically downregulate E-cadherin expression and significantly increase MTA1 expression (Fig. 1B).
Silencing E-cadherin induces migration ability of $2 B 4$ cells. To determine whether E-cadherin affects the migration ability of 2B4 cells, a wound healing assay was conducted. The wound healing ability of cells reflects their movement and migration on the surface on which they are anchored to for growth. At 0,12 and $24 \mathrm{~h}$ after wounding, transfected E-cadherin siRNA cells demonstrated an increased level of wound healing compared with the mock and control siRNA cells (Fig. 2). At $24 \mathrm{~h}$ after wounding, the wound of the E-cadherin siRNA transfected group had healed, while that of the cells from the control siRNA and mock groups had not closed $(\mathrm{P}<0.01$ compared with the control cells).

Silencing E-cadherin alters the malignant phenotype of $2 B 4$ cells. The 2B4 cells were transfected with siRNA against E-cadherin for $48 \mathrm{~h}$ and a solid-phase adhesion assay was used to detect the alteration in cell adhesion ability. For the cells 
A

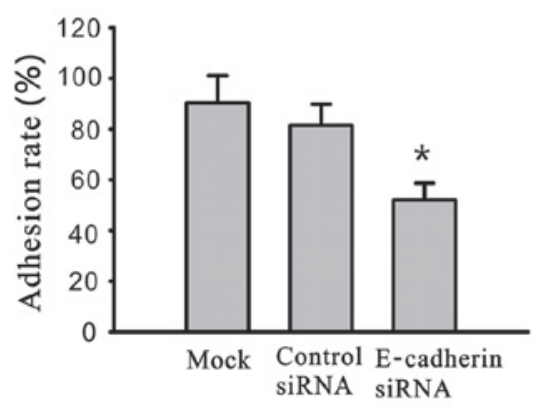

B

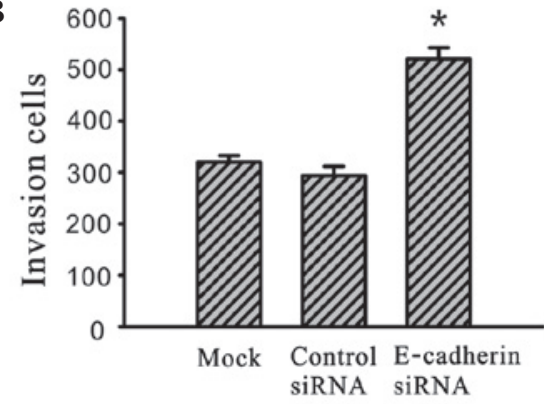

C

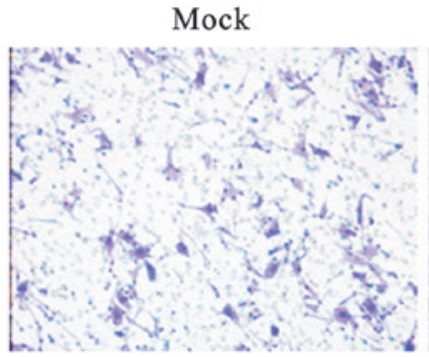

Control siRNA

E-cadherin siRNA
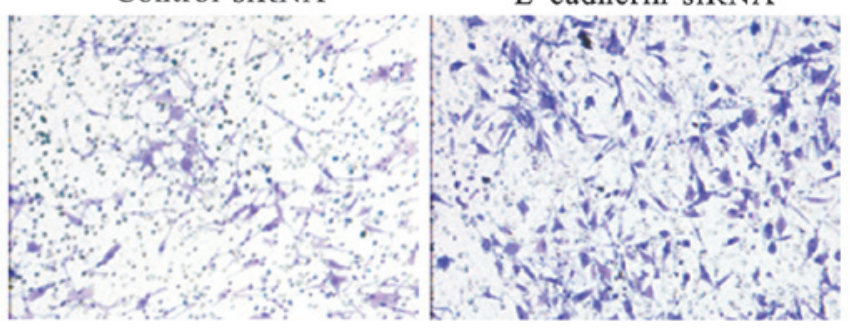

D
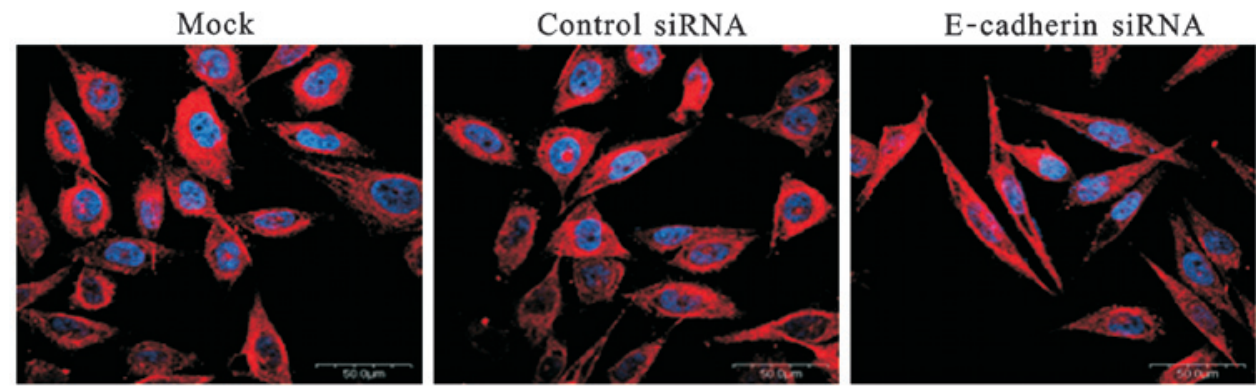

Figure 3. Downregulation of E-cadherin affects cell adhesion, invasion ability and cytoskeleton construction. Cells were transfected with E-cadherin siRNA or negative control siRNA for $48 \mathrm{~h}$, and the adhesive ability was examined by MTT assay. All experiments were repeated three times. (A) The adhesion ability to solid phase was significantly downregulated in cells treated with E-cadherin siRNA. ${ }^{*} \mathrm{P}<0.05$ compared with non-treated cells (mock) and negative control siRNA transfected cells. (B) Invasion ability was assessed using the Matrigel ${ }^{\mathrm{TM}}$-coated transwell system. $8 \times 10^{3}$ cells/well were placed in a Matrigel-coated Boyden chamber and allowed to invade for $24 \mathrm{~h}$. Graph reveals the quantification of invasive cells from the lower side of the transwell inserts. "P $<0.05$ compared with non-treated cells (mock) and negative control siRNA transfected cells. (C) Representative images of invasion experiments. (D) The altered structure (600-fold) of the cytoskeleton was detected using confocal microscopy. Red fluorescent staining represents the $\alpha$-tublin and blue staining represents the nucleus. siRNA, small interfering RNA; MTT, 3-(4,5-dimethylthiazol-2-yl)-2,5-diphenyltetrazolium bromide.

A

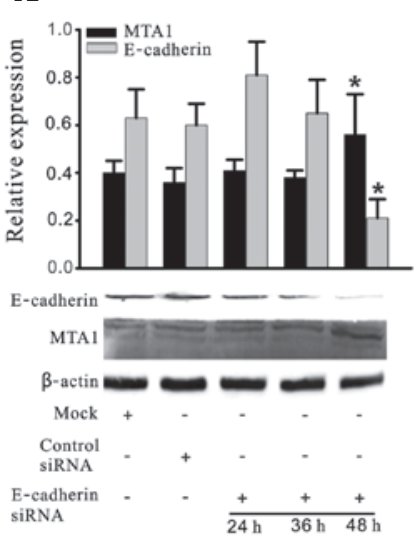

B

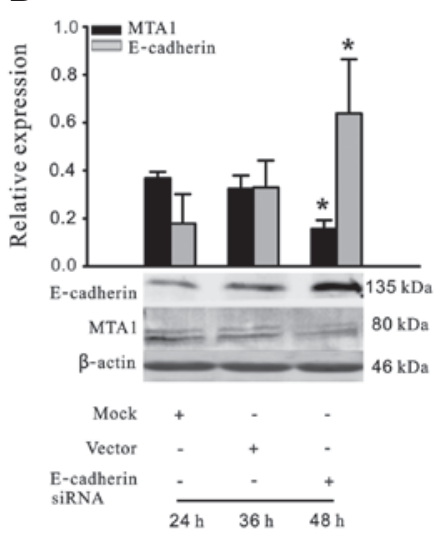

Figure 4. E-cadherin regulates MTA1 expression. (A) Cells were transfected with E-cadherin siRNA or negative control siRNA for 24,36 or $48 \mathrm{~h}$. Western blot analysis demonstrated that E-cadherin siRNA increased MTA1 expression at $48 \mathrm{~h}$. (B) Cells were transfected with E-cadherin full length plasmid for 24, 36 or $48 \mathrm{~h}$. Western blot analysis demonstrated that MTA1 expression was decreased at $48 \mathrm{~h}$. The changes were quantified in the upper panels. " $\mathrm{P}<0.05$ compared with negative control siRNA transfection. All experiments were repeated three times. MTA1, metastasis-associated gene 1; siRNA, small interfering RNA.

with a similar concentration of surface-coated FN, silencing of E-cadherin by siRNA significantly inhibited the adhesion process compared with the mock and control siRNA treated cells (Fig. 3A). 2B4 cells were transfected with siRNA against E-cadherin for $48 \mathrm{~h}$ and a Matrigel-coated transwell model was used to detect the alteration in cell invasion ability. The number of cells at the lower side of the membrane reflects the invasion ability of the cells being investigated. The number of cells that invaded to the lower side of the membrane were $320.22 \pm 12.50$, $293.56 \pm 18.32$ and $521.13 \pm 21.67$ for the mock, control siRNAtreated and E-cadherin-siRNA-treated cells, respectively. Compared with the mock and control-siRNA treated group, the invasion ability of the cells treated with E-cadherin siRNA was significantly greater (Fig. $3 \mathrm{~B}$ and $=\mathrm{C}$ ). Each assay was repeated three times. The decreased adhesion ability as well as an increased invasion and migration ability, influences a change in the architecture and composition of the cytoskeleton. To examine whether the inhibition of E-cadherin expression affects the subcellular distribution and organization of the cytokeratin filaments, immunofluorescence analyses were conducted using E-cadherin siRNA-transfected, control-transfected siRNA 


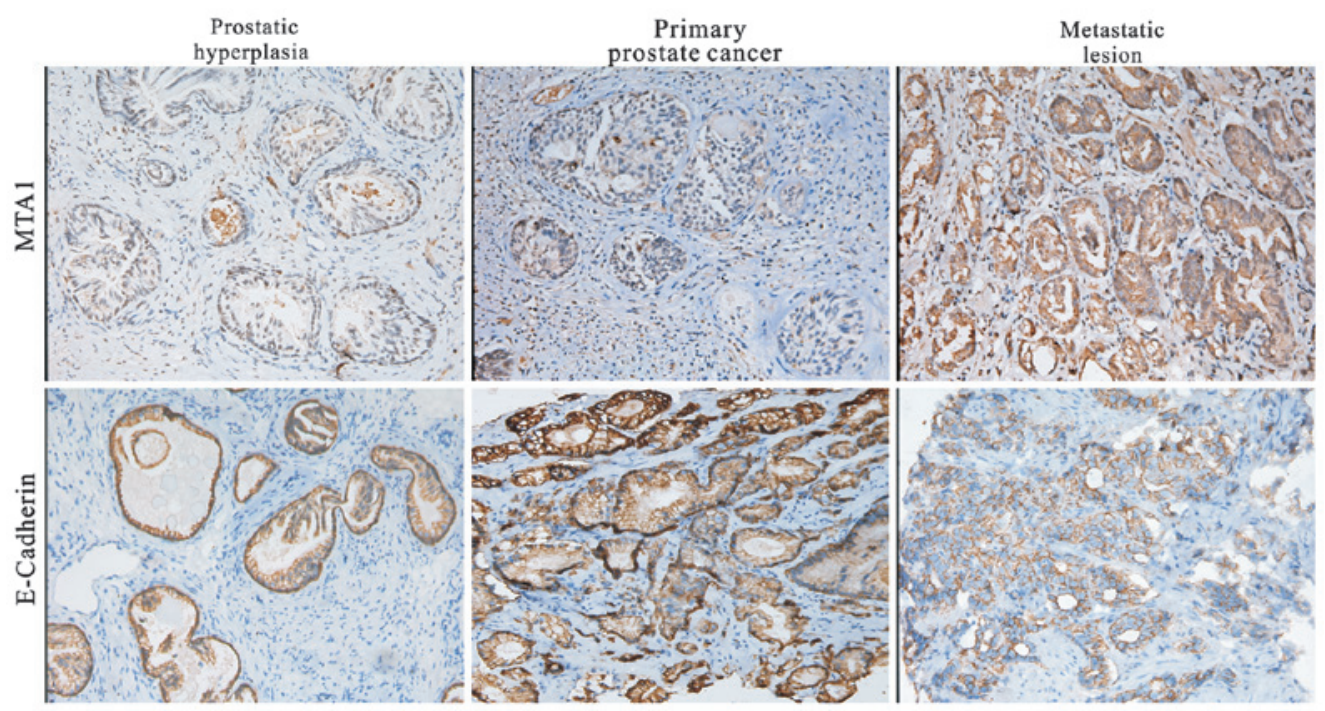

Figure 5. Expression of E-cadherin and MTA1 in prostate cancer tissues. Typical results for E-cadherin and MTA1 staining determined by immunohistochemistry was observed in $\mathrm{BPH}$, localized prostate cancer and metastatic prostate cancer. Magnification, x200. MTA1, metastasis-associated gene 1; BPH, benign prostatic hyperplasia.

and mock cells. In the E-cadherin siRNA-transfected cells we observed an increased elongated spindle-shape morphology with extended pseudopodia between adjacent cells and an increase in cellular polarity strength compared with the control siRNA and mock cells (Fig. 3D). These results suggest that E-cadherin plays a key role in controling the malignant phenotypes of 2B4 cells, including the adhesion ability, invasion ability and cytoskeleton organization.

E-cadherin regulates MTA1 expression. The $2 \mathrm{~B} 4$ cells were treated with E-cadherin siRNA and full length E-cadherin plasmid for various time periods between 24 and $48 \mathrm{~h}$, and western blot analyis was used to detect E-cadherin and MTA1 protein expression. As demonstrated in Fig. 4A, $48 \mathrm{~h}$ after treatment with E-cadherin siRNA, E-cadherin expression was significantly downregulated, while MTA1 expression was significantly upregulated. Additionally, following empty vector pCMV-SPORT6 transfection, MTA1 expression was significantly downregulated and E-cadherin was significantly upregulated $48 \mathrm{~h}$ after treatment (Fig. 4B). All experiments were repeated three times.

Orientation of E-cadherin and MTA1 protein in prostate tissue. E-cadherin expression was detected predominantly in the cytoplasm and membrane of the prostate cancer tissues. MTA1 protein positive signaling was detected in the cytoplasm or nucleus of cancer cells. A representative result of the immunohistochemistry for MTA1 and E-cadherin in prostate cancer is shown in Fig. 5. The samples probed with E-cadherin antibody demonstrated $2+$ or $3+$ membrane staining of the epithelial cells. Cytoplasmic staining was $0-$ or $1+$. The positive staining in the prostate lesions had distinct circumferential E-cadherin immunoreactivity of high intensity, which revealed that E-cadherin is a cell membrane protein.

Correlation between expression of E-cadherin and MTAI in prostate carcinoma tissue. To further assess whether
E-cadherin expression levels correlate with the expression of MTA1 in prostate cancer metastasis, we analyzed the results for E-cadherin and MTA1 immunoreactivity. Intact E-cadherin immunostaining was observed in $70 \%(14 / 20)$ of benign prostatic hyperplasia (BPH), 53.6\% (15/28) of primary cancer and $16.7 \%(2 / 12)$ of metastatic lesion tissues. MTA1 expression levels in BPH, primary cancer and metastatic lesion tissues were 30 (6/20), 75 (21/28) and 25\% (3/12), respectively. E-cadherin expression was decreased in the prostate cancer tissues compared with the BPH tissues $(\mathrm{P}<0.001)$, while MTA expression was increased in the prostate cancer tissues compared with the BPH tissues $(\mathrm{P}<0.05$; Table I). Furthermore, we analyzed the correlation between the expression levels of E-cadherin and MTA1 in the tumor group. As shown in Table II, we observed a statistically significant correlation between E-cadherin and MTA1 immunoreactivity in the prostate cancer group $\left(\mathrm{P}<0.05 ; \mathrm{r}_{\mathrm{s}}=-0.434\right.$, respectively).

\section{Discussion}

Cancer metastasis is a complex and multi-step process that commonly leads to the mortality of cancer patients. Cell adhesion molecules play an important role in the development of recurrent, invasive and distant metastasis in the process of cancer progression (6). E-cadherin is a key cell-to-cell adhesion molecule, which plays a significant role in the invasion and metastasis of tumor cells (10). In the majority of, if not all, cancers of epithelial origin, the loss of cell-to-cell adhesion mediated by E-cadherin may occur concomitantly with the progression towards tumor malignancy (10).

The majority of previous studies indicate that E-cadherin has a close correlation with metastasis and invasion of a number of tumors, including ovarian $(13)$, breast $(34,35)$, pancreatic (36), gastric (37) and prostate cancer $(38,39)$. With regard to prostate cancer, E-cadherin expression in the cancer cells at metastasis in lymph node sites is lower than 
Table I. Expression of E-cadherin and MTA1 in prostate carcinoma tissue.

\begin{tabular}{|c|c|c|c|c|c|c|c|}
\hline \multirow[b]{2}{*}{ Group } & \multirow{2}{*}{$\begin{array}{l}\text { No. of } \\
\text { cases }\end{array}$} & \multicolumn{2}{|c|}{ E-cadherin } & \multirow[b]{2}{*}{ P-value } & \multicolumn{2}{|c|}{ MTA1 } & \multirow[b]{2}{*}{ P-value } \\
\hline & & - & + & & - & + & \\
\hline BPH & 20 & 6 & 14 & 0.044 & 14 & 6 & 0.028 \\
\hline Tumor & 40 & 23 & 17 & & 16 & 24 & \\
\hline Primary cancer & 28 & 13 & 15 & 0.030 & 7 & 21 & 0.003 \\
\hline Metastatic lesion & 12 & 10 & 2 & & 9 & 3 & \\
\hline
\end{tabular}

MTA1, metastasis-associated gene 1; BPH, benign prostatic hyperplasia.

Table II. Correlation between immunohistochemically detected expression of MTA1 and E-cadherin in prostate cancer.

\begin{tabular}{lrrrrr}
\hline & \multicolumn{2}{c}{ MTA1 } & & & \\
\cline { 2 - 3 } E-cadherin & \multicolumn{1}{c}{} & + & Total & P-value & $\mathrm{r}_{\mathrm{s}}$ \\
\hline- & 5 & 18 & 23 & 0.005 & -0.434 \\
+ & 11 & 6 & 17 & & \\
Total & 16 & 24 & 40 & & \\
\hline
\end{tabular}

MTA1, metastasis-associated gene 1.

in the primary prostate cancer (38). Studies have suggested that prostate cancer cells with low expression of E-cadherin are more invasive (40); therefore, the absence of E-cadherin expression in prostate cancer predicts the potential of metastasis to the bone compared with prostate cancer that does express E-cadherin (41). A recent study suggested that upregulating E-cadherin expression by valproic acid inhibits prostate cancer cell migration (42). The effects of E-cadherin on the malignant phenotype of prostate cancer cells and possible molecular mechanisms are not entirely understood.

MTA1 is a highly expressed with high potential to metastasize in a number of cancer types (22-24). Earlier studies have demonstrated an inverse correlation between the levels of MTA1 and E-cadherin, and identified that increased MTA1 expression is associated with increased invasiveness and reduced expression of E-cadherin in tumor cells $(23,43)$. However, studies with regard to the expression and correlation of E-cadherin and MTA1 in prostate cancer have rarely been reported. In this study, we demonstrate that the loss of E-cadherin is able to induce metastasis of prostate cancer cells through upregulated MTA1 expression.

Downregulated expression of E-cadherin in prostate cancer and upregulated expression of MTA1 is consistent with previous studies $(38,41,44)$. Therefore, it was hypothesized that there is a certain inherent connection between E-cadherin and MTA1, and their coordination may lead to tumor cell invasion and metastasis. E-cadherin may be upregulated by MTA1 siRNA in melanoma cells, which was also confirmed in our previous study in cervical cancer cells $(29,30)$. MTA1 overexpression resulted in the downregulation of E-cadherin expression in ovarian cancer (23).
In the present study, E-cadherin protein was markedly expressed in 2B4 (poorly-metastatic prostate adenocarcinoma cell lines) cells and weakly expressed in 1E8 (highly-metastatic prostate adenocarcinoma cell lines) cells. To clarify the molecular characteristics of E-cadherin, E-cadherin siRNA was transfected into $2 \mathrm{~B} 4$ cells for $48 \mathrm{~h}$. Our results revealed that MTA1 expression was increased when E-cadherin expression was decreased. E-cadherin downregulation is one of the principal events during EMT and a frequently reported phenomena in embryonic development and cancer progression $(45,46)$. MTA1 has been suggested to determine EMT phenotype, mainly through the downregulated expression of E-cadherin, which leads to EMT (28). Developing cancer cells acquire migratory features concomitant with a loss of E-cadherin expression during carcinogenesis $(13,47)$. We identified that downregulation of E-cadherin expression resulted in the promotion of $2 \mathrm{~B} 4$ cell migration and invasion, and the inhibition of adhesion capability via upregulated MTA1 in vitro.

The increased adhesion ability and increased invasion and migration ability is accompanied by a change in the architecture and composition of the cytoskeleton. EMT is a key step toward cancer metastasis, and E-cadherin is regarded as a main indicator of the epithelial/mesenchymal phenotype switching (19). E-cadherin loss is suggestive of EMT, and tumor cell invasion and metastasis are associated with EMT $(10,48)$. Cell changes, including morphological and gene expression alterations, are necessary for EMT $(49,50)$. In the present study, cells acquired an elongated spindle-shape morphology with extended pseudopodia between adjacent cells due to a decrease in E-cadherin and an increase in cellular polarity strength. Our results indicate that E-cadherin may play an important role in the cellular polarity of prostate cancer cells. We identified that the protein level of MTA1 was upregulated when E-cadherin was decreased. We also identified that expression of E-cadherin regulates MTA1 expression in 2B4 cells treated with E-cadherin siRNA or full length E-cadherin plasmid at different times (24-48 h). Our data suggest that E-cadherin regulates MTA1 in a time-dependent manner.

To further investigate the expression levels of and correlation between E-cadherin and MTA1 in prostate cancer, we examined BPH, carcinoma in situ and metastatic carcinoma tissues using immunohistochemical staining. Our results demonstrated that there is an inverse correlation between protein expression of E-cadherin and MTA1 in prostate cancer. 
Loss of E-cadherin expression in $57.5 \%$ of prostate cancer tissues in association with the increase of MTA expression in $60 \%$ of tissues, suggests that the two proteins are closely related to prostate cancer progression. This result suggests the possibility that E-cadherin and MTA1 act together as prognosis predictors of metastasis and progression of prostate cancer. A combined testing strategy for detecting MTA1 and E-cadherin may be sufficient for selecting high-risk patients with metastasis.

We revealed that loss of E-cadherin-induced MTA1 expression in prostate cancer $2 \mathrm{~B} 4$ cells promotes the migration and invasion of $2 \mathrm{~B} 4$ cells. Our results provide a new insight into the mechanisms of E-cadherin regulation of MTA1 in prostate cancer, and suggests that E-cadherin may combine with MTA1 and alter the malignant phenotype of prostate cancer. A combined testing strategy for detecting MTA1 and E-cadherin may be sufficient for selecting high-risk patients with metastasis. E-cadherin and MTA1 may be potential powerful targets for the treatment of various types of cancer.

\section{Acknowledgements}

This study was supported by grants from the Major State Basic Research Development Program of China (973 Program, 2009; No. CB521808) and the National Science Foundation of China (Nos. 30700895, 30770913, 81001006, 30901587 and 81101971).

\section{References}

1. Siegel R, Ward E, Brawley O and Jemal A: Cancer statistics, 2011: the impact of eliminating socioeconomic and racial disparities on premature cancer deaths. CA Cancer J Clin 61: 212-236, 2011

2. Boyle P and Ferlay J: Cancer incidence and mortality in Europe, 2004. Ann Oncol 16: 481-488, 2005.

3. Mundy GR: Metastasis to bone: causes, consequences and therapeutic opportunities. Nat Rev Cancer 2: 584-593, 2002.

4. Logothetis CJ and Lin SH: Osteoblasts in prostate cancer metastasis to bone. Nat Rev Cancer 5: 21-28, 2005.

5. Eccles SA and Welch DR: Metastasis: recent discoveries and novel treatment strategies. Lancet 369: 1742-1757, 2007.

6. Okegawa T, Pong RC, Li Y and Hsieh JT: The role of cell adhesion molecule in cancer progression and its application in cancer therapy. Acta Biochim Pol 51: 445-457, 2004.

7. Bogenrieder T and Herlyn M: Axis of evil: molecular mechanisms of cancer metastasis. Oncogene 22: 6524-6536, 2003.

8. Cavallaro U and Dejana E: Adhesion molecule signalling: not always a sticky business. Nat Rev Mol Cell Biol 12: 189-197, 2011.

9. Huber MA, Kraut N and Beug H: Molecular requirements for epithelial-mesenchymal transition during tumor progression. Curr Opin Cell Biol 17: 548-558, 2005.

10. Cavallaro U and Christofori G: Cell adhesion and signalling by cadherins and Ig-CAMs in cancer. Nat Rev Cancer 4: 118-132, 2004.

11. Hanahan D and Weinberg RA: Hallmarks of cancer: the next generation. Cell 144: 646-674, 2011

12. Berx G and van Roy F: Involvement of members of the cadherin superfamily in cancer. Cold Spring Harb Perspect Biol 1: a003129, 2009.

13. Sawada K, Mitra AK, Radjabi AR, et al: Loss of E-cadherin promotes ovarian cancer metastasis via alpha 5-integrin, which is a therapeutic target. Cancer Res 68: 2329-2339, 2008.

14. Onder TT, Gupta PB, Mani SA, Yang J, Lander ES and Weinberg RA: Loss of E-cadherin promotes metastasis via multiple downstream transcriptional pathways. Cancer Res 68: 3645-3654, 2008.

15. Singh A and Settleman J: EMT, cancer stem cells and drug resistance: an emerging axis of evil in the war on cancer. Oncogene 29: 4741-4751.
16. Yilmaz M and Christofori G: EMT, the cytoskeleton, and cancer cell invasion. Cancer Metastasis Rev 28: 15-33, 2009.

17. Moreno-Bueno G, Portillo F and Cano A: Transcriptional regulation of cell polarity in EMT and cancer. Oncogene 27: 6958-6969, 2008.

18. Gravdal K, Halvorsen OJ, Haukaas SA and Akslen LA: A switch from $\mathrm{E}$-cadherin to $\mathrm{N}$-cadherin expression indicates epithelial to mesenchymal transition and is of strong and independent importance for the progress of prostate cancer. Clin Cancer Res 13: 7003-7011, 2007.

19. Liu YN, Liu Y, Lee HJ, Hsu YH and Chen JH: Activated androgen receptor downregulates E-cadherin gene expression and promotes tumor metastasis. Mol Cell Biol 28: 7096-7108, 2008.

20. Toh Y, Pencil SD and Nicolson GL: A novel candidate metastasis-associated gene, mta1, differentially expressed in highly metastatic mammary adenocarcinoma cell lines. cDNA cloning, expression, and protein analyses. J Biol Chem 269: 22958-22963, 1994.

21. Manavathi B, Peng S, Rayala SK, et al: Repression of Six3 by a corepressor regulates rhodopsin expression. Proc Natl Acad Sci USA 104: 13128-13133, 2007.

22. Qian H, Lu N, Xue L, et al: Reduced MTA1 expression by RNAi inhibits in vitro invasion and migration of esophageal squamous cell carcinoma cell line. Clin Exp Metastasis 22: 653-662, 2005.

23. Dannenmann C, Shabani N, Friese K, Jeschke U, Mylonas I and Bruning A: The metastasis-associated gene MTA1 is upregulated in advanced ovarian cancer, represses ERbeta, and enhances expression of oncogenic cytokine GRO. Cancer Biol Ther 7: 1460-1467, 2008.

24. Ryu SH, Chung YH, Lee H, et al: Metastatic tumor antigen 1 is closely associated with frequent postoperative recurrence and poor survival in patients with hepatocellular carcinoma. Hepatology 47: 929-936, 2008.

25. Nicolson GL, Nawa A, Toh Y, Taniguchi S, Nishimori K and Moustafa A: Tumor metastasis-associated human MTA1 gene and its MTA1 protein product: role in epithelial cancer cell invasion, proliferation and nuclear regulation. Clin Exp Metastasis 20: 19-24, 2003

26. Toh Y and Nicolson GL: The role of the MTA family and their encoded proteins in human cancers: molecular functions and clinical implications. Clin Exp Metastasis 26: 215-227, 2009.

27. Pakala SB, Singh K, Reddy SD, et al: TGF- $\beta 1$ signaling targets metastasis-associated protein 1, a new effector in epithelial cells. Oncogene 30: 2230-2241.

28. Radaelli E, Damonte P and Cardiff RD: Epithelial-mesenchymal transition in mouse mammary tumorigenesis. Future Oncol 5: 1113-1127, 2009.

29. Qian H, Yu J, Li Y, et al: RNA interference of metastasis-associated gene 1 inhibits metastasis of B16F10 melanoma cells in a C57BL/6 mouse model. Biol Cell 99: 573-581, 2007.

30. Rao Y, Wang H, Fan L and Chen G: Silencing MTA1 by RNAi reverses adhesion, migration and invasiveness of cervical cancer cells $(\mathrm{SiHa})$ via altered expression of $\mathrm{p} 53$, and E-cadherin $/ \beta$-catenin complex. J Huazhong Univ Sci Technolog Med Sci 31: 1-9, 2011.

31. Hunt NC, Douglas-Jones AG, Jasani B, Morgan JM and Pignatelli M: Loss of E-cadherin expression associated with lymph node metastases in small breast carcinomas. Virchows Arch 430: 285-289, 1997.

32. Manavathi B and Kumar R: Metastasis tumor antigens, an emerging family of multifaceted master coregulators. J Biol Chem 282: 1529-1533, 2007.

33. Liu Y, Zheng J, Fang W, et al: Isolation and characterization of human prostate cancer cell subclones with different metastatic potential. Zhonghua Bing Li Xue Za Zhi 28: 361-364, 1999 (In Chinese).

34. Chao YL, Shepard CR and Wells A: Breast carcinoma cells re-express E-cadherin during mesenchymal to epithelial reverting transition. Mol Cancer 9: 179, 2010.

35. Kowalski PJ, Rubin MA and Kleer CG: E-cadherin expression in primary carcinomas of the breast and its distant metastases. Breast Cancer Res 5: R217-R222, 2003.

36. von Burstin J, Eser S, Paul MC, et al: E-cadherin regulates metastasis of pancreatic cancer in vivo and is suppressed by a SNAIL/HDAC1/HDAC2 repressor complex. Gastroenterology 137: 361-371, 2009.

37. Tang B, Peng ZH, Yu PW, Yu G and Qian F: Expression and significance of $\mathrm{Cx} 43$ and E-cadherin in gastric cancer and metastatic lymph nodes. Med Oncol 28: 502-508, 2011. 
38. Cheng L, Nagabhushan M, Pretlow TP, Amini SB and Pretlow TG: Expression of E-cadherin in primary and metastatic prostate cancer. Am J Pathol 148: 1375-1380, 1996.

39. Rubin MA, Mucci NR, Figurski J, Fecko A, Pienta KJ and Day ML: E-cadherin expression in prostate cancer: a broad survey using high-density tissue microarray technology. Hum Pathol 32: 690-697, 2001.

40. Mao Q, Zheng X, Yang K, et al: Suppression of migration and invasion of $\mathrm{PC} 3$ prostate cancer cell line via activating E-cadherin expression by small activating RNA. Cancer Invest 28: 1013-1018, 2010.

41. Pontes J Jr, Srougi M, Borra PM, Dall' Oglio MF, Ribeiro-Filho LA and Leite KR: E-cadherin and beta-catenin loss of expression related to bone metastasis in prostate cancer. Appl Immunohistochem Mol Morphol 18: 179-184, 2010.

42. Zhang L, Wang G, Wang L, Song C, Wang X and Kang J: Valproic acid inhibits prostate cancer cell migration by up-regulating E-cadherin expression. Pharmazie 66: 614-618, 2011.

43. Zhang H, Stephens LC and Kumar R: Metastasis tumor antigen family proteins during breast cancer progression and metastasis in a reliable mouse model for human breast cancer. Clin Cancer Res 12: 1479-1486, 2006.

44. Hofer MD, Kuefer R, Varambally S, et al: The role of metastasis-associated protein 1 in prostate cancer progression. Cancer Res 64: 825-829, 2004
45. Thiery JP: Epithelial-mesenchymal transitions in tumour progression. Nat Rev Cancer 2: 442-454, 2002.

46. Polyak K and Weinberg RA: Transitions between epithelial and mesenchymal states: acquisition of malignant and stem cell traits. Nat Rev Cancer 9: 265-273, 2009.

47. Richmond PJ, Karayiannakis AJ, Nagafuchi A, Kaisary AV and Pignatelli M: Aberrant E-cadherin and alpha-catenin expression in prostate cancer: correlation with patient survival. Cancer Res 57: 3189-3193, 1997.

48. Di Croce L and Pelicci PG: Tumour-associated hypermethylation: silencing E-cadherin expression enhances invasion and metastasis. Eur J Cancer 39: 413-414, 2003.

49. Maeda M, Johnson KR and Wheelock MJ: Cadherin switching: essential for behavioral but not morphological changes during an epithelium-to-mesenchyme transition. J Cell Sci 118: 873-887, 2005.

50. Christofori G: New signals from the invasive front. Nature 441: 444-450, 2006 